\title{
The Full Database of Countries with Potential COVID-19 Data Misreport based on Benford's Law
}

\author{
${ }^{1}$ Ahmad Kilani and ${ }^{2}$ Georgios P. Georgiou
}

${ }^{1}$ Department of Comparative Politics, RUDN University, Moscow, Russia

${ }^{2}$ Department of Languages and Literature, University of Nicosia, Nicosia, Cyprus

\begin{abstract}
The aim of this database is to provide researchers and scholars a unified database for potential data misreport by 171 countries regarding their COVID-19 daily reported cases. The analysis employs three different tests (chi-square, Kuiper, and Mean Absolute Deviation) to determine if the data given by each country in the world fit Benford's Law.
\end{abstract}

\section{Benford's law}

Benford's Law (BL) is extensively used to test the legitimacy of data in numerous applications, such as in finance, economics, and politics. BL indicates that the first digit of a naturally transpire decimal number is more likely to be equal to 1 , and the possibilities of the first digit to be equal to the subsequent numbers, decrease progressively.

The formula of BL is as follows, where $D$ represents the digits between 1 and 9 and $\mathrm{P}$ is the probability:

$$
P(d)=\log _{10}(d+1)-\log _{10} d=\log _{10}\left(1+\frac{1}{d}\right), \text { for }(d=1, \ldots, 9)
$$

Using this formula, the expected frequencies for digits in first position are illustrated below:

\begin{tabular}{llllllllll}
\hline Digit & $\mathbf{1}$ & $\mathbf{2}$ & $\mathbf{3}$ & $\mathbf{4}$ & $\mathbf{5}$ & $\mathbf{6}$ & $\mathbf{7}$ & $\mathbf{8}$ & $\mathbf{9}$ \\
\hline $\begin{array}{l}\text { Expected } \\
\text { Frequency }\end{array}$ & .30103 & .17609 & .12494 & .09691 & .07918 & .06695 & .05799 & .05115 & .04576 \\
\hline
\end{tabular}

\section{Tests}

To measure the difference between the observed and expected first digit BL distribution for a country we used a chi-square test, which is based on the formula below: 


$$
\chi^{2}=\sum \frac{(O i-E i)^{2}}{E i}
$$

Where $O i$ is the observed and $E i$ is the expected absolute frequencies for digit $i$.

The Kuiper test (a modified Kolmogorov-Smirnov test) (Kuiper 1960) ignores sample size and it is based on the following formula:

$$
V=\left(D_{n}^{+}+D_{n}^{-}\right)(\sqrt{n}+0.155+0,24 \sqrt{n})
$$

where $D_{n}^{+}=\sup \left[\mathrm{F}\left(\mathrm{O}_{\mathrm{i}}\right)-\mathrm{F}\left(\mathrm{E}_{\mathrm{i}}\right)\right]$ and $D_{n}^{-}=\sup \left[\mathrm{F}\left(\mathrm{E}_{\mathrm{i}}\right)-\mathrm{F}\left(\mathrm{O}_{\mathrm{i}}\right)\right]$, and $\mathrm{F}($.$) stands for cumulated$ relative frequencies. The discrepancy statistics $D^{+}$and $D^{-}$characterize the absolute sizes of the differences between the two distributions being compared: the absolute and the observed distribution.

The Mean Absolute Deviation (MAD) test that also ignores sample size yet if the data sample size is small it may be inclined to false positives errors, when the results conclude nonconformity from unbiased data (Nigrini 2012). Henceforth, Nirgini's MAD can only be applied to large sample size i.e. the total daily reported cases in the world. Johnson and Weggenmann (2013) have adjusted the MAD calculation method to better solve the false positive problem; hence, an adjusted MAD can be used for country's data set. The MAD statistic is calculated as follows:

$$
\text { Mean Absolute Deviation }=\frac{1}{N} \sum_{1}^{N} f i[X i-\bar{X}]
$$

Where $X i$ is the difference between actual occurrence rate and Benford occurrence rate and $\bar{X}$ is the mean of the difference between actual occurrence rate and Benford occurrence rate. Whereas in Nigrini's MAD, $X i$ is the actual occurrence rate and $\bar{X}$ is the Benford occurrence rate. The absolute symbol means that the deviation is given a positive sign irrespective of whether it is positive or negative. Individual differences are then totaled and divided by 9 (the number of leading digits) to yield the mean absolute deviation (Johnson and Weggenmann 2013).

\section{Data collection}

We used publicly available data from the European Centre for Disease Prevention and Control website $^{1}$ on reported daily cases of Covid-19 in the world starting from the first case of Covid-19 in each country to the $12^{\text {nd }}$ of November 2020.

The below Figure shows the Benfordness of the World's reported daily cases where the blue bars represent the world's observed daily reported cases and the intermittent red line represents BL.

\footnotetext{
${ }^{1}$ https://www.ecdc.europa.eu/en
} 


\section{Digits Distribution}

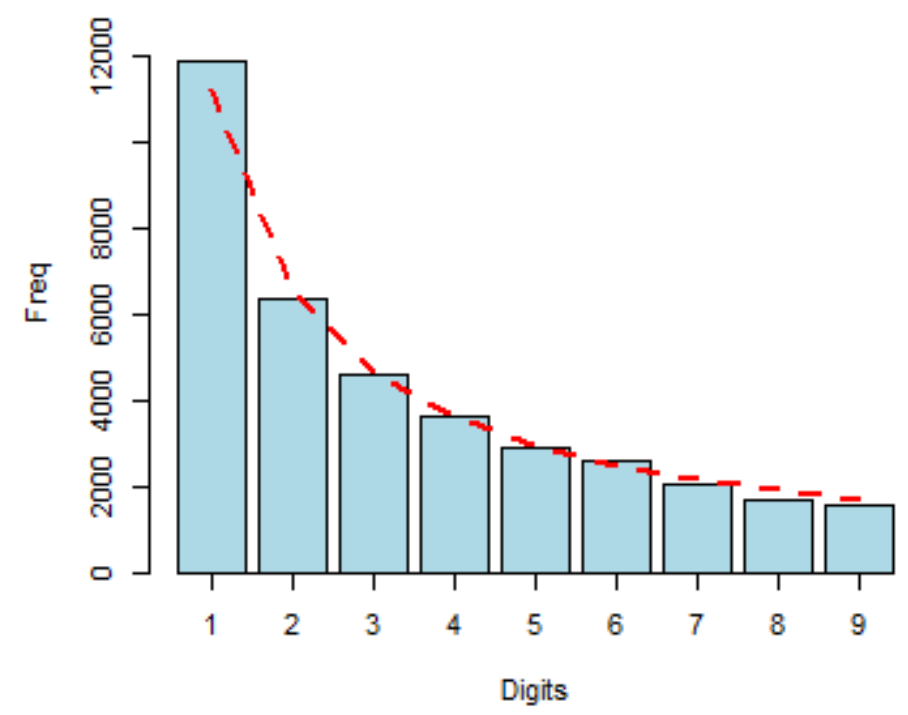

\section{The database}

The ranges of the tests are shown below. If the value of the chi-square and the Kuiper tests are more than 20.09 and 2 respectively, it is considered that a country's distribution curve does not fit BL. Similarly, in the MAD test, if the value is more than 0.015 , the curve does not conform to BL.

\begin{tabular}{lllll}
$\boldsymbol{\alpha}$ level & chi-square & Kuiper & MAD & MAD conformity ranges \\
0.01 & $>15.51$ & $>1.65$ & $>0.006$ & Close conformity \\
0.05 & $15.51-17.54$ & $1.65-1.75$ & $0.006-0.012$ & Acceptable conformity \\
0.1 & $17.54-20.09$ & $1.75-2.0$ & $0.012-0.015$ & Marginally acceptable conformity \\
\hline none & $<20.09$ & $<2$ & $<0.015$ & Nonconformity \\
\hline
\end{tabular}




\section{Countries}

Afghanistan

Albania

Algeria

Andorra

Angola

Argentina

Armenia

Australia

Austria

Azerbaijan

Bahrain

Bangladesh

Belarus

Belgium

Belize

Benin

Bhutan

Bolivia

Bosnia and Herzegovina

Botswana

Brazil

Brunei Darussalam

Bulgaria

Burkina Faso

\section{Chi-square Kuiper MAD $^{2}$}

\begin{tabular}{|lll}
8.00 & 1.49 & 0.013 \\
19.02 & 2.98 & 0.035 \\
12.05 & 1.84 & 0.021 \\
7.99 & 1.46 & 0.016
\end{tabular}

6.51

0.95

0.014

21.77

3.31

0.039

8.80

1.65

0.013

4.94

1.26

0.014

6.29

1.52

0.014

13.51

1.31

0.023

30.62

3.97

0.041

26.13

3.83

0.040

66.84

3.43

0.037

8.72

2.04

0.019

12.78

1.25

0.021

11.56

1.09

0.015

23.15

2.33

0.033

14.22

2.41

0.026

9.65

1.87

0.023

13.26

1.05

0.021

17.72

2.83

0.026

43.49

1.50

0.051

8.35

1.97

0.020

9.58

1.67

0.023 


\begin{tabular}{|c|c|c|c|}
\hline Burundi & 7.37 & 1.01 & 0.015 \\
\hline Cambodia & 37.80 & 2.38 & 0.048 \\
\hline Cameroon & 5.96 & 1.06 & 0.017 \\
\hline Canada & 7.65 & 1.85 & 0.013 \\
\hline Cape Verde & 9.77 & 1.53 & 0.015 \\
\hline Central African Republic & 4.85 & 0.87 & 0.015 \\
\hline Chad & 5.87 & 1.18 & 0.012 \\
\hline Chile & 23.92 & 3.00 & 0.032 \\
\hline China & 6.39 & 1.65 & 0.013 \\
\hline Colombia & 42.10 & 3.89 & 0.041 \\
\hline Comoros & 21.38 & 1.29 & 0.030 \\
\hline Congo & 6.55 & 0.69 & 0.017 \\
\hline Costa Rica & 14.48 & 2.59 & 0.031 \\
\hline Croatia & 5.71 & 1.01 & 0.016 \\
\hline Cuba & 17.37 & 2.89 & 0.031 \\
\hline Cyprus & 6.16 & 1.65 & 0.014 \\
\hline Czech Republic & 1.46 & 0.61 & 0.009 \\
\hline Denmark & 6.18 & 1.13 & 0.014 \\
\hline Djibouti & 5.07 & 1.09 & 0.011 \\
\hline Dominican Republic & 8.31 & 1.77 & 0.020 \\
\hline East Timor & 126.28 & 2.04 & 0.096 \\
\hline Ecuador & 13.27 & 2.14 & 0.019 \\
\hline Egypt & 41.88 & 4.62 & 0.051 \\
\hline El Salvador & 9.94 & 0.99 & 0.019 \\
\hline Equatorial Guinea & 18.09 & 1.41 & 0.027 \\
\hline
\end{tabular}




\begin{tabular}{|c|c|c|c|}
\hline Eritrea & 11.04 & 1.17 & 0.021 \\
\hline Estonia & 4.50 & 0.70 & 0.008 \\
\hline Ethiopia & 7.46 & 1.68 & 0.013 \\
\hline Finland & 9.48 & 2.12 & 0.020 \\
\hline France & 6.95 & 1.65 & 0.013 \\
\hline Gabon & 16.28 & 2.03 & 0.030 \\
\hline Gambia & 12.91 & 1.43 & 0.021 \\
\hline Georgia & 5.90 & 0.72 & 0.013 \\
\hline Germany & 2.42 & 0.52 & 0.009 \\
\hline Ghana & 4.44 & 1.00 & 0.011 \\
\hline Greece & 11.87 & 2.07 & 0.018 \\
\hline Guatemala & 10.31 & 1.91 & 0.015 \\
\hline Guinea & 20.05 & 2.78 & 0.030 \\
\hline Guinea-Bissau & 19.47 & 1.67 & 0.031 \\
\hline Guyana & 3.80 & 0.96 & 0.011 \\
\hline Haiti & 4.12 & 1.21 & 0.009 \\
\hline Honduras & 18.37 & 3.16 & 0.031 \\
\hline Hungary & 8.73 & 1.20 & 0.016 \\
\hline Iceland & 4.03 & 1.21 & 0.010 \\
\hline India & 26.49 & 3.58 & 0.035 \\
\hline Indonesia & 26.39 & 2.93 & 0.030 \\
\hline Iran & 56.76 & 4.51 & 0.046 \\
\hline Iraq & 36.47 & 4.25 & 0.044 \\
\hline Ireland & 2.20 & 1.02 & 0.011 \\
\hline Israel & 3.78 & 1.23 & 0.011 \\
\hline
\end{tabular}




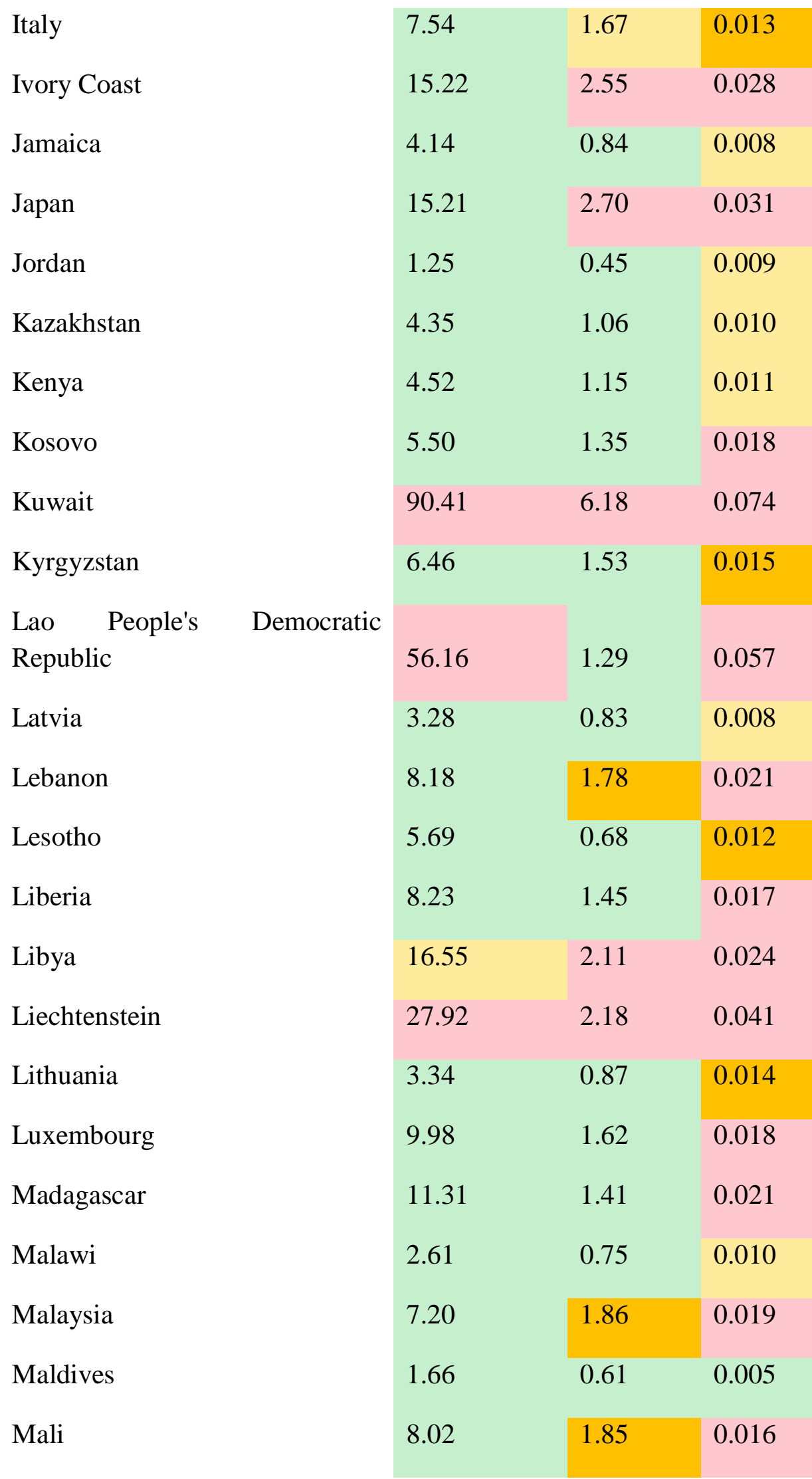




\begin{tabular}{|c|c|c|c|}
\hline Malta & 5.30 & 1.38 & 0.015 \\
\hline Mauritania & 5.45 & 1.09 & 0.014 \\
\hline Mauritius & 20.34 & 1.34 & 0.034 \\
\hline Mexico & 33.06 & 3.34 & 0.036 \\
\hline Moldova & 3.16 & 0.67 & 0.013 \\
\hline Mongolia & 36.55 & 2.35 & 0.045 \\
\hline Montenegro & 5.79 & 1.00 & 0.011 \\
\hline Morocco & 8.30 & 1.96 & 0.018 \\
\hline Mozambique & 6.63 & 1.52 & 0.018 \\
\hline Myanmar & 13.83 & 2.18 & 0.026 \\
\hline Namibia & 3.89 & 1.09 & 0.015 \\
\hline Nepal & 7.89 & 1.75 & 0.017 \\
\hline Netherlands & 12.66 & 2.39 & 0.022 \\
\hline New Zealand & 7.56 & 1.44 & 0.016 \\
\hline Nicaragua & 43.15 & 1.94 & 0.049 \\
\hline Niger & 7.65 & 1.25 & 0.015 \\
\hline Nigeria & 7.96 & 1.37 & 0.010 \\
\hline North Macedonia & 17.89 & 2.68 & 0.029 \\
\hline Norway & 2.96 & 0.97 & 0.012 \\
\hline Oman & 5.83 & 1.52 & 0.020 \\
\hline Pakistan & 11.13 & 2.12 & 0.018 \\
\hline Palestine & 33.47 & 3.87 & 0.045 \\
\hline Panama & 44.52 & 4.04 & 0.041 \\
\hline Papua New Guinea & 17.16 & 1.26 & 0.029 \\
\hline Paraguay & 11.35 & 2.21 & 0.019 \\
\hline
\end{tabular}




\begin{tabular}{|c|c|c|c|}
\hline Peru & 28.98 & 3.39 & 0.041 \\
\hline Philippines & 21.62 & 3.04 & 0.030 \\
\hline Poland & 23.74 & 3.25 & 0.036 \\
\hline Portugal & 14.25 & 2.78 & 0.029 \\
\hline Qatar & 40.65 & 3.68 & 0.039 \\
\hline Romania & 11.81 & 2.35 & 0.022 \\
\hline Russia & 60.91 & 4.79 & 0.057 \\
\hline Rwanda & 2.00 & 0.65 & 0.008 \\
\hline Saudi Arabia & 23.32 & 2.69 & 0.028 \\
\hline Senegal & 14.81 & 2.61 & 0.029 \\
\hline Serbia & 7.45 & 1.52 & 0.018 \\
\hline Sierra Leone & 4.11 & 1.19 & 0.013 \\
\hline Singapore & 4.70 & 1.50 & 0.016 \\
\hline Slovakia & 5.06 & 1.50 & 0.015 \\
\hline Slovenia & 7.27 & 1.62 & 0.016 \\
\hline Somalia & 3.99 & 0.91 & 0.014 \\
\hline South Africa & 11.45 & 2.20 & 0.024 \\
\hline South Korea & 1.99 & 0.73 & 0.008 \\
\hline South Sudan & 4.11 & 0.71 & 0.012 \\
\hline Spain & 5.44 & 0.92 & 0.012 \\
\hline Sri Lanka & 2.69 & 0.67 & 0.007 \\
\hline Sudan & 5.48 & 0.97 & 0.012 \\
\hline Suriname & 8.05 & 1.18 & 0.012 \\
\hline Swaziland & 1.64 & 0.50 & 0.006 \\
\hline Sweden & 11.71 & 2.05 & 0.018 \\
\hline
\end{tabular}




\begin{tabular}{|c|c|c|c|}
\hline Switzerland & 1.42 & 0.48 & 0.008 \\
\hline Syria & 33.29 & 2.83 & 0.034 \\
\hline Taiwan & 32.18 & 2.93 & 0.040 \\
\hline Tajikistan & 163.73 & 6.43 & 0.095 \\
\hline Tanzania & 24.03 & 0.69 & 0.032 \\
\hline Thailand & 8.89 & 1.24 & 0.015 \\
\hline $\begin{array}{l}\text { The Democratic Republic Of } \\
\text { The Congo }\end{array}$ & 4.80 & 1.22 & 0.015 \\
\hline Togo & 8.13 & 1.77 & 0.017 \\
\hline Trinidad and Tobago & 2.34 & 0.67 & 0.005 \\
\hline Tunisia & 7.94 & 1.61 & 0.017 \\
\hline Turkey & 72.28 & 5.78 & 0.067 \\
\hline Uganda & 15.25 & 2.43 & 0.027 \\
\hline Ukraine & 18.84 & 2.82 & 0.029 \\
\hline United Arab Emirates & 5.72 & 1.61 & 0.016 \\
\hline United Kingdom & 4.82 & 1.07 & 0.011 \\
\hline United States & 19.84 & 3.41 & 0.033 \\
\hline Uruguay & 3.92 & 1.02 & 0.012 \\
\hline Uzbekistan & 21.29 & 3.15 & 0.033 \\
\hline Venezuela & 11.51 & 1.59 & 0.017 \\
\hline Vietnam & 6.91 & 1.35 & 0.014 \\
\hline Yemen & 15.11 & 1.42 & 0.018 \\
\hline Zambia & 3.60 & 0.64 & 0.013 \\
\hline Zimbabwe & 8.20 & 1.42 & 0.017 \\
\hline
\end{tabular}




\section{References}

Johnson, G.G., and J. Weggenmann. 2013. "Exploratory research applying Benford's Law to selected balances in the financial statements of state governments." Academy of Accounting and Financial Studies Journal 17(3): 31-44.

Kuiper, N. H. 1960. "Tests concerning random points on a circle. $\mathrm{P}$, ." roceedings of the Koninklijke Nederlandse Akademie van Wetenschappen Series A. 63 (pp. 38-47).

Nigrini, Mark J. 2012. Benford's Law: Applications for Forensic Accounting, Auditing, and Fraud Detection. New Jersey: Wiley Corporate F\&A. 